\title{
La propagation de la rage par des chiens en provenance du Nunavut
}

\author{
Curry PS ${ }^{*}$, Kostiuk $\mathrm{D}^{2}$, Werker $\mathrm{DH}^{1}$, Baikie $\mathrm{M}^{3}$, Ntiamoah $\mathrm{W}^{4}$, Atherton $\mathrm{F}^{5}$, Enns $\mathrm{A}^{6}$, Opondo $\mathrm{J}^{6}$, \\ Guirgis $\mathrm{H}^{7}$, Mema $\mathrm{S}^{8}$
}

\section{Résumé}

Contexte : Les enquêtes sur les animaux atteints de la rage qui franchissent les frontières provinciales ou territoriales sont complexes et exigeantes sur le plan des ressources, car de nombreux secteurs et administrations gouvernementales doivent y participer.

Objectif : Décrire les réponses intergouvernementales mises en œuvre à la suite de la découverte de deux chiots non apparentés atteints de la rage, qui provenaient du Nunavut.

Méthodologie : Sommaire descriptif des enquêtes mises en œuvre à la suite de l'identification d'un premier chiot atteint de la rage en Alberta (août 2013), puis d'un second en Saskatchewan (décembre 2014).

Résultats : Les services de santé publique et d'agriculture de cinq provinces et territoires, en plus de l'Agence canadienne d'inspection des aliments ( $\mathrm{ACIA})$, ont participé à ces enquêtes. En Alberta, un chiot devenu malade après avoir été transporté par avion du Nunavut a dû être euthanasié; un diagnostic de rage (variante du renard arctique) a par la suite été posé. Dix-huit personnes ont été examinées pour déterminer si elles avaient été exposées à la rage; une prophylaxie antirabique post-exposition a été administrée à neuf d'entre elles. Un chien domestique qui avait été exposé au chiot a aussi été euthanasié, mais le test de dépistage de la rage s'est révélé négatif. Au Nunavut, la mère du chiot enragé et les autres chiots de la portée ont été mis en quarantaine. En Saskatchewan, un autre chiot est tombé malade durant son transport par avion en provenance du Nunavut. Le chiot a par la suite été euthanasié et un diagnostic de rage (variante du renard arctique) a été posé. Une prophylaxie antirabique post-exposition a été administrée à deux des trois personnes qui avaient été exposées à I'animal en Saskatchewan, dont un technicien vétérinaire, ainsi qu'à deux résidents de la Nouvelle-Écosse qui avaient été exposés au chiot pendant leur séjour au Nunavut. Un vaccin de rappel a été administré à un chien domestique, qui a été mis en quarantaine pendant 45 jours et est demeuré asymptomatique. II a toutefois été impossible de retracer la mère de ce chiot atteint de la rage et les autres chiots de la portée au Nunavut. Dans les deux cas, il est probable que l'origine de la rage ait été une exposition à un renard arctique.

Conclusion : Le déplacement de chiens en provenance du Nord, là où la rage du renard arctique est endémique, pose un risque pour la santé humaine et animale et pourrait nuire au contrôle de la rage au Canada. II n'existe à l'heure actuelle aucun cadre national pour prévenir le transfert d'animaux susceptibles d'être atteints de la rage entre les provinces et territoires du Canada.

\author{
Affiliations \\ ${ }^{1}$ Ministère de la Santé de la \\ Saskatchewan, Direction de la \\ santé de la population, Regina \\ (Saskatchewan) \\ ${ }^{2}$ Ministère de l'Agriculture et \\ des Forêts de l'Alberta, Direction \\ de la santé animale, Edmonton \\ (Alberta) \\ ${ }^{3}$ Ministère de la Santé et des \\ Services sociaux du Nunavut, \\ lqaluit (Nunavut) \\ ${ }^{4}$ Ministère de la Santé et des \\ Services sociaux du Nunavut, \\ Cambridge Bay (Nunavut) \\ ${ }^{5}$ Ministère de la Santé et \\ Mieux-être de la Nouvelle-Écosse, \\ Halifax (Nouvelle-Écosse) \\ ${ }^{6}$ Région sanitaire de Saskatoon, \\ Saskatoon (Saskatchewan) \\ ${ }^{7}$ Santé publique de l'Alberta, \\ Services de la santé publique \\ environnementale, Edmonton \\ (Alberta) \\ ${ }^{8}$ Santé publique de l'Alberta, \\ Calgary (Alberta) (services \\ actuellement regroupés avec \\ I'Interior Health Authority de la \\ Colombie-Britannique)
}

*Correspondance : pcurry@ health.gov.sk.ca

Citation proposée : Curry PS, Kostiuk D, Werker DH, Baikie M, Ntiamoah W, Atherton F, et al. La propagation de la rage par des chiens en provenance du Nunavut. Relevé des maladies transmissibles au Canada 2016;42:135-9. https://doi.org/10.14745/ccdr.v42i06a02f

\section{Introduction}

Entre 2013 et 2014, un diagnostic de rage a été posé pour deux chiots non apparentés en provenance du Nunavut, après leur arrivée dans leur province d'accueil respective; ces chiots étaient tous deux âgés de moins d'un an et avaient été adoptés séparément. Le premier chiot est arrivé à Calgary, en Alberta, en passant par Edmonton, en Alberta, en août 2013, et le second est arrivé dans une région rurale de la Saskatchewan en décembre 2014, après un arrêt à Yellowknife, aux Territoires du Nord-Ouest, et à Edmonton, en Alberta. Cinq provinces et territoires (Nunavut, Territoires du Nord-Ouest, Alberta, Saskatchewan et Nouvelle-Écosse), ainsi que l'Agence canadienne d'inspection des aliments (ACIA), ont participé aux enquêtes sur l'exposition des humains et des animaux. Deux résidents de la Nouvelle-Écosse ont été exposés alors 
qu'ils travaillaient au Nunavut. Le rapport sur cette éclosion illustre la complexité de ces enquêtes, en raison de leur caractère multidisciplinaire et du nombre de partenaires qui doivent y participer, et fait ressortir les défis et les lacunes dans le domaine de la prévention et du contrôle de la rage à l'échelle nationale.

\section{Méthodologie}

Le diagnostic et la déclaration de deux cas confirmés de rage chez des chiots ont déclenché la tenue de deux enquêtes de santé publique ayant pour but de retracer et d'évaluer les humains et les animaux qui auraient pu être exposés à la rage. Ces enquêtes visaient à prévenir la propagation de la rage aux humains et aux animaux. L'ACIA s'est chargée des enquêtes sur les animaux jusqu'au 21 mars 2013 et a participé à l'enquête menée en Alberta. En Saskatchewan, I'enquête sur les animaux a été prise en charge par le ministère de l'Agriculture de la province. Au Nunavut et dans les Territoires du Nord-Ouest, les enquêtes sur les animaux ont été confiées à l'ACIA ou aux autorités territoriales de santé publique, tandis que les autorités régionales, provinciales et territoriales de santé publique ont été chargées de toutes les enquêtes touchant la santé humaine.

Les employés des services régionaux, provinciaux et territoriaux de la santé publique et de l'agriculture qui ont directement participé à ces deux enquêtes ont passé en revue leurs dossiers et en ont fait des résumés qui ont été intégrés au présent rapport par trois auteurs (PSC, DK, DHW). Un rapport préalablement publié sur le chiot de l'Alberta (1) a également été mis à jour à partir de l'information obtenue du Nunavut et des dossiers de l'Alberta. L'équipe d'enquête a ensuite passé en revue les résumés descriptifs pour s'assurer de leur exactitude.

\section{Résultats}

\section{Alberta, 2013}

En juin 2013, une résidente de Calgary qui travaillait dans une communauté éloignée du Nunavut y a remarqué la présence d'une famille de chiots qui fouillaient dans les ordures pour se nourrir. Elle voulait ramener un des chiots chez elle, mais ne le pouvait pas car les chiots étaient encore trop jeunes pour être séparés de leur mère. Quelques semaines plus tard, un de ses amis s'est rendu dans cette communauté du Nunavut et lui a rapporté un chiot. Le chiot, un husky âgé de cinq mois, a été transporté par avion du Nunavut à Calgary, après une escale à Edmonton.

Le 9 juillet 2013, peu après son arrivée, le chiot a été amené dans une clinique vétérinaire de Calgary pour un premier examen et une première série de vaccins. La propriétaire a déclaré que le chiot se portait bien et qu'il avait le comportement normal d'un chiot errant qui avait été adopté.

Le chiot a été ramené à la clinique vétérinaire pour une seconde série de vaccins le 8 août 2013; il a alors été décidé de reporter l'administration du vaccin antirabique, car le chiot devait recevoir un vaccin contre Bordetella. Le chiot s'adaptait bien à sa nouvelle vie et sa propriétaire a indiqué que le chiot était heureux et calme et que son entraînement à la propreté progressait bien.
Cependant, quatre jours plus tard, soit le 12 août 2013, la propriétaire a ramené le chiot à la clinique vétérinaire parce qu'il avait commencé à vomir et qu'il attaquait et mordait son autre chien. Le chiot avait également mordu le colocataire de la propriétaire à l'arrière de la jambe, mais la morsure n'avait pas transpercé complètement l'épiderme. Le chiot hurlait de façon intermittente et sa propriétaire n'arrivait pas à le calmer. Le chiot a été amené à la clinique vétérinaire dans une cage; une perche a été utilisée pour sortir le chiot de la cage, d'abord pour le museler, puis pour l'euthanasier. Le vétérinaire n'a pas fait d'examen clinique du chiot à ce stade, car les signes cliniques laissaient fortement présumer qu'il s'agissait de la rage. Le vétérinaire a consulté le vétérinaire de district de l'ACIA qui lui a expliqué qu'un chien non vacciné, chez qui l'on soupçonnait la rage, devait être mis en quarantaine pendant six mois ou être euthanasié et testé pour la rage. La propriétaire a choisi de faire euthanasier le chiot et de lui faire subir un test de dépistage de la rage.

L'ACIA a envoyé les échantillons au laboratoire d'expertise de la rage de Lethbridge, en Alberta, le 15 août 2013. Le laboratoire a déclaré que le test de l'absorption fluorescente des anticorps avait été positif. Le typage effectué par la suite a révélé qu'il s'agissait du virus rabique de la variante du renard arctique.

L'ACIA a aussi été consultée au sujet de la prise en charge du chien d'un an de la propriétaire qui avait été mordu par le chiot. Ce chien avait été vacciné contre la rage lorsqu'il était plus jeune, mais il n'avait pas reçu le vaccin de rappel à un an; il a donc été classé comme un animal primo-vacciné. Il a été recommandé que ce chien reçoive immédiatement un vaccin de rappel, puis qu'il soit soumis à une période d'observation de 45 jours ou qu'il soit euthanasié conformément au protocole de I'ACIA. La propriétaire a choisi de faire euthanasier son chien; le test de dépistage de la rage s'est révélé négatif.

Le bureau de district d'Edmonton de l'ACIA, qui était responsable des maladies animales à déclaration obligatoire au Nunavut, a communiqué avec le propriétaire des chiots et a délivré une ordonnance de quarantaine visant les autres chiots vivants. Peu après la délivrance de l'avis de quarantaine, deux chiots ont été éliminés pour des raisons non liées à la rage. Tous deux étaient en santé au moment de leur élimination. La mère et les deux chiots restants de la portée étaient toujours en santé à la fin de la période de quarantaine de six mois. Bien que le propriétaire n'ait fait mention d'aucun contact entre ses chiens et des renards arctiques, les chiens étaient gardés à l'extérieur et, cet hiver-là, on avait découvert dans la communauté un renard arctique qui a par la suite réagi positivement au test de dépistage de la rage.

Les responsables de la santé publique de l'Alberta et du Nunavut ont évalué les expositions de 18 personnes qui avaient été en contact avec le chiot; ils ont considéré que neuf d'entre elles avaient eu des expositions à haut risque et ces personnes ont reçu une prophylaxie post-exposition. Quatre membres du ménage ont aussi reçu une prophylaxie post-exposition, de même que 4 des 12 personnes qui connaissaient le propriétaire du chiot et un des vétérinaires l'ayant soigné. Une personne a reçu sa dernière dose du vaccin antirabique en Nouvelle-Écosse. 


\section{Saskatchewan, 2014 à 2015}

Au début de décembre 2014, un chiot de moins d'un an ressemblant à un husky s'était aventuré sur un chantier de construction situé dans une communauté éloignée du Nunavut. Le chiot, qui semblait en bonne santé, a été recueilli et pris en charge par plusieurs travailleurs du chantier. L'un des travailleurs a par la suite décidé d'adopter le chiot et de le ramener en Saskatchewan. Le 16 décembre 2014, le chiot a été transporté par avion, du Nunavut à Yellowknife, aux Territoires du Nord-Ouest, puis à bord d'un autre vol à destination d'Edmonton (Alberta). Le propriétaire a ensuite voyagé à bord d'un véhicule privé pour se rendre dans une communauté rurale située dans la région sanitaire de Saskatoon.

Durant le trajet en voiture, le chiot a commencé à être malade et à afficher d'importants changements dans son comportement, et il a mordu un membre de la famille. Le lendemain, le propriétaire a amené le chien dans une clinique vétérinaire de la région. Dans son rapport, le vétérinaire a indiqué que le chiot avait les yeux vitreux, qu'il salivait abondamment et qu'il était très dysphorique, aboyant et rejetant la tête vers l'avant et l'arrière. Comme la rage faisait partie du diagnostic différentiel, le chiot a été euthanasié et sa tête a été envoyée au laboratoire de l'ACIA pour analyse. Une enquête de santé publique a été entreprise; les résultats de cette enquête sont résumés ci-après.

Le vétérinaire de la clinique a informé le vétérinaire responsable de l'évaluation du risque de rage du ministère de l'Agriculture de la Saskatchewan de la possibilité que le chiot soit atteint de la rage. Ce vétérinaire a informé les services de santé publique de la région sanitaire de Saskatoon, ainsi que le ministère de la Santé et le ministère de l'Agriculture de la province, de la présence possible d'un animal atteint de la rage. La région sanitaire de Saskatoon a procédé à une évaluation préliminaire du risque d'exposition chez les humains. Deux collègues du propriétaire avaient été exposés au chiot alors qu'ils travaillaient au Nunavut et ils étaient depuis retournés en Nouvelle-Écosse. La morsure dont avait été victime le membre de la famille a causé une plaie punctiforme sur le bras qui a été traitée adéquatement dans le cabinet d'un médecin. Un technicien vétérinaire, qui avait déjà reçu le vaccin contre la rage en préexposition, s'est coupé avec le couteau utilisé pour décapiter le chiot, après que le couteau eut été plongé dans l'eau de Javel.

Le 19 décembre, I'ACIA a fait savoir que le test de l'absorption fluorescente des anticorps était positif. Le vétérinaire responsable de l'évaluation du risque de rage a informé les responsables de la santé publique et le ministère de l'Agriculture de ces résultats. Le typage réalisé par la suite a confirmé la présence du virus de la rage de la variante du renard arctique.

Le 19 décembre, le ministère de l'Agriculture et le ministère de la Santé de la Saskatchewan ont tous deux informé les autorités albertaines de la découverte d'un chiot atteint de la rage. Le ministère de la Santé de la Saskatchewan en a aussi informé les autorités de la santé publique du Nunavut, des Territoires du Nord-Ouest et de la Nouvelle-Écosse. Le propriétaire du chiot avait déjà informé ses collègues de travail de la maladie du chien, et ces derniers s'étaient présentés aux services de santé publique de la Nouvelle-Écosse et avaient déjà commencé une prophylaxie antirabique post-exposition.
Le membre de la famille qui avait été mordu par le chiot a été évalué pour déterminer s'il avait été exposé à la rage, et une prophylaxie antirabique post-exposition lui a été administrée. Cette personne avait prévu partir en voyage et elle a donné suite à ses projets; la quatrième et dernière dose du traitement prophylactique lui a donc été administrée en Irlande (les dispositions à cette fin avaient été réglées en collaboration avec le point focal national du Règlement sanitaire international en Irlande). Deux autres membres de la famille ont été évalués, mais il a été établi qu'ils n'avaient pas été exposés à la rage. Le technicien vétérinaire a reçu deux doses de rappel du vaccin. Des échantillons de sang ont été prélevés de tous les employés de la clinique pour déterminer leurs titres d'anticorps antirabiques.

Les responsables de la santé publique de l'Alberta et des Territoires du Nord-Ouest ont évalué les travailleurs des aéroports qui auraient pu être en contact avec le chiot durant son transport en provenance du Nunavut. Le chiot avait été gardé en cage pendant les vols. II n'y a eu aucune exposition professionnelle. Les responsables de la santé publique de l'Alberta ont également confirmé qu'aucun autre humain n'avait été exposé durant le transport du chiot par voie terrestre, d'Edmonton vers son nouveau domicile en Saskatchewan.

Au Nunavut, les responsables de la santé publique ont mené une enquête auprès de toutes les personnes de la communauté qui auraient pu être exposées. Personne n'a reçu de prophylaxie antirabique post-exposition. Un message d'intérêt public a été diffusé pour informer les membres de la communauté de la situation concernant la rage et leur recommander d'éviter les chiens errants et de signaler tout chien ayant un comportement inhabituel.

Comme il n'y a aucun service vétérinaire ou agricole au Nunavut, l'enquête sur les animaux a été menée par les responsables de la santé publique. Il a été impossible de retracer, au Nunavut, la mère du chiot atteint de la rage et les autres chiots de la portée. Cependant, le 25 décembre 2014, un jeune chien husky, qui présentait de la bave mousseuse près de la bouche, a été découvert errant à proximité de la même communauté. Le chien a été abattu et les tests effectués par la suite se sont révélés positifs à la rage de la variante du renard arctique. À peu près à la même période, un renard abattu dans la région s'est lui aussi révélé positif à la rage de la variante du renard arctique. Aucune exposition humaine à cet animal n'a été recensée.

Le propriétaire du chiot atteint de la rage avait un autre chien. Ce chien avait déjà été vacciné contre la rage, mais son statut vaccinal n'était pas à jour. Le ministère de l'Agriculture de la Saskatchewan a exigé que le chien reçoive un vaccin antirabique de rappel et qu'il soit mis en quarantaine pendant 45 jours par le propriétaire. Le chien est demeuré en bonne santé durant la période de quarantaine, et aucun autre suivi n'a été exigé.

Les enquêtes n'ont révélé aucun autre animal qui avait été exposé au chiot atteint de la rage.

Dans les deux cas, les infections chez les humains ont été évitées, et la rage ne s'est propagée à aucun autre animal domestique, outre les deux chiots. La rage de la variante du renard arctique est endémique dans bon nombre de communautés du Grand Nord. Les chiens y sont exposés lorsqu'ils viennent en contact 
avec des renards arctiques enragés ou avec d'autres animaux qui ont été infectés par ces renards.

Quelque 25 employés des services de santé publique et d'agriculture ont participé directement à chacune de ces enquêtes. Ce nombre n'inclut pas les ressources qui ont été nécessaires pour la prestation des soins aux humains et aux animaux, les analyses de laboratoire ou les communications opérationnelles et publiques dans l'ensemble des secteurs touchés.

\section{Discussion}

Ces deux enquêtes constituent les deuxième et troisième rapports faisant état du déplacement de chiens enragés au Canada depuis 2012. Des incidents similaires avaient été signalés au Québec en 2012 (2). Ces cas font ressortir le risque que le transfert de chiots en provenance du Nord (où la rage du renard arctique est endémique) présente pour la santé humaine et animale, ainsi que les conséquences négatives que cela peut avoir sur le contrôle de la rage au Canada. Ces deux enquêtes montrent également que les personnes qui vivent dans d'autres régions du Canada, où la rage est plus répandue chez les mouffettes, les chauves-souris et les ratons laveurs, ne sont pas toujours conscientes du risque élevé de rage chez les chiens en provenance du Nunavut et d'autres régions du Nord du Canada. En 2013 et 2014, 23 \% et $27 \%$ des animaux atteints de la rage au Nunavut et dans les Territoires du Nord-Ouest étaient des chiens domestiques (3).

Les chiens non vaccinés, en provenance du Nunavut et des Territoires du Nord-Ouest, devraient être considérés comme étant à haut risque d'être atteints de la rage, et les humains ayant été fortement exposés à ces animaux selon les enquêtes devraient recevoir une prophylaxie antirabique post-exposition sans attendre les résultats des analyses. En raison de la période d'incubation relativement longue (et variable) de la rage chez les chiens (en moyenne de 3 à 12 semaines), il pourrait arriver que des chiens en apparence en bonne santé soient transportés hors de la région alors qu'ils sont infectés $(4,5)$. Les chiots posent un risque particulier, car ils sont attirants et les humains souhaitent souvent les adopter à une période où ils n'ont pas encore été vaccinés contre la rage (6). Dans ces deux enquêtes, des animaux domestiques ont également été exposés, mais aucun n'a été infecté. Il convient toutefois de souligner qu'un de ces chiens a peut-être été euthanasié durant la période d'incubation et qu'il aurait donc pu être atteint de la rage s'il avait vécu. De plus, le transfert de ces chiots errants vers des régions où vivent également des renards roux pose le risque d'introduire ou de réintroduire la rage de la variante du renard arctique chez les espèces fauniques de ces régions.

Lorsque des animaux enragés traversent des frontières provinciales ou territoriales, la complexité des enquêtes augmente, et ceci peut retarder la rapidité des interventions requises pour prévenir et contrôler la propagation de la rage. Comme l'évaluation du risque de rage chez les animaux et le prélèvement des échantillons animaux pour les tests de dépistage de la rage en laboratoire ne relèvent plus de la responsabilité de l'ACIA, chaque province et territoire a mis en place son propre programme contre la rage, et cela a donné lieu à l'établissement d'un ensemble de programmes disparates, sans protocoles opérationnels clairement définis pour coordonner les enquêtes intergouvernementales. L'absence de protocole national normalisé peut retarder la notification des autres provinces et territoires ce qui, en retour, peut retarder l'identification et l'évaluation du risque d'exposition chez les humains ou les animaux domestiques - des mesures pourtant essentielles pour prévenir et maîtriser la rage. En Saskatchewan, l'enquête sur le chiot a incité le ministère de la Santé de cette province à élaborer un schéma de processus illustrant les différentes étapes des enquêtes lors de la découverte d'animaux susceptibles d'avoir la rage, à définir les facteurs exigeant la déclaration immédiate de tout cas de rage au Ministère et à mettre en place un formulaire intergouvernemental normalisé pour la déclaration des cas d'exposition chez les animaux (7).

Le Canada exige la présentation d'une preuve de vaccination récente contre la rage pour les animaux exotiques ou domestiques qui sont importés en provenance d'autres pays (8). Cependant, il n'existe à l'heure actuelle aucune exigence en matière de vaccination, ni aucun cadre stratégique national, pour prévenir le mouvement d'animaux susceptibles d'être atteints de la rage entre les provinces et territoires du Canada. Un tel cadre pourrait toutefois permettre d'éviter des situations comme celles provoquées par le transfert de ces deux chiots atteints de la rage. Ce cadre pourrait énoncer un ensemble de lois, de règlements, de politiques ou d'orientations exigeant la vaccination contre la rage des animaux domestiques qui traversent des frontières provinciales ou territoriales.

À cette fin, il faudrait obtenir la collaboration et l'engagement de divers intervenants, notamment l'industrie du transport aérien et le secteur des transports, l'industrie du tourisme, les autorités responsables des parcs, les associations de piégeage et de chasse, les pourvoiries et les groupes de protection des animaux, pour élaborer des politiques et du matériel de communication visant à favoriser le respect des exigences relatives à la vaccination des animaux transportés. Ầ titre d'exemple, les organismes de protection des animaux pourraient établir des politiques exigeant que les chiens rescapés soient vaccinés et examinés par un vétérinaire avant d'être adoptés. Des interventions sont également nécessaires pour améliorer la couverture vaccinale des chiens domestiques dans le Nord et ainsi protéger les résidents du Nord et du Sud; ces interventions pourraient consister par exemple en l'embauche de vaccinateurs non spécialisés pour tenir de vastes cliniques ou campagnes de vaccination, en l'élargissement du programme de vaccinateurs non spécialisés à d'autres régions, ainsi qu'en la tenue de cliniques de vaccination élargies avec l'aide de vétérinaires $d^{\prime}$ 'autres provinces ou territoires. Ces mesures permettraient non seulement de réduire le risque de propagation de la rage vers les régions du Sud, mais amélioreraient également la prévention et le contrôle de la rage dans le Nord du Canada.

\section{Conclusion}

Les mouvements d'humains et d'animaux entre les provinces et territoires du Canada peuvent facilement entraîner la propagation de la rage vers des régions où la maladie est moins répandue. Une approche nationale, prévoyant la vaccination antirabique obligatoire des animaux transportés entre des provinces ou territoires du Canada, l'adoption de pratiques exemplaires de gestion et une plus grande sensibilisation des Canadiens au caractère endémique de la rage dans le Nord, 
pourrait être intégrée à un programme national de prévention et de contrôle de la rage. Sans un tel programme, le Canada restera vulnérable à la propagation de la rage entre les provinces et territoires.

\section{Remerciements}

Les auteurs aimeraient remercier les personnes et les organisations suivantes pour leur contribution à ces enquêtes : Betty Althouse, Wendy Wilkins et Clarence Bischop (vétérinaire responsable de l'évaluation du risque de rage), ministère de l'Agriculture de la Saskatchewan - Division de la santé animale; Lisa Haubrich, ministère de la Santé de la Saskatchewan; André Corriveau, ministère de la Santé et des Services sociaux des Territoires du Nord-Ouest; Jack Muggaberg, Agence canadienne d'inspection des aliments; Francis Nkemamin Ndemafia, Santé publique de l'Alberta; ainsi que le Health Protection Surveillance Centre, Health Service Executive, d'Irlande.

\section{Conflit d'intérêts}

Aucun.

\section{Références}

1. Mema S, Friesen B, Desai S, Rock M, Mclntyre L. Rabies in a Calgary puppy adopted from the Arctic. Can J Public Health; 104(7)e510.

2. Abedelaziz N, Gravel G, Markowski F, Leblanc MA. Vigie - Interventions : Rage chez des chiots. Flash Vigie 2012; 7(2):Fév 2012.

3. Canadian Food Inspection Agency (CFIA). Rabies in Canada. Ottawa ON: CFIA; 2016. (Disponible en français : http:// www.inspection.gc.ca/animaux/animaux-terrestres/maladies/ declaration-obligatoire/rage/cas-de-rage-au-canada/fra/1356 156989919/1356157139999).

4. Greene CE, Rupprect CE. Rabies and other Lyssavirus infections. In: Greene CE, editor. Infectious diseases of the dog and cat. Philadelphia: WB Saunders; 2006. p. 167-183.

5. Fekadu M. Canine rabies. In: Baer GM editor. The natural history of rabies. Boca Ratton: CDC Press: 1991. p. 368-375.

6. White J, Taylor SM, Wolfram KL, O'Connor B. Rabies in a 10week old puppy. Can Vet. J 2007;48(9):931-934.

7. Saskatchewan Ministry of Health. Inter-jurisdictional referral following an animal exposure. In: Section 4: Vector-borne and zoonotic disease. Regina SK: Government of Saskatchewan; 2014. http://www.ehealthsask.ca/services/manuals/ Documents/cdc-section-4.pdf\#page=26.

8. Canadian Food Inspection Agency (CFIA). Import and travel requirements. Ottawa ON: CFIA; 2016. http://www. inspection.gc.ca/animals/terrestrial-animals/imports/policies/ live-animals/pets/eng/1326600389775/1326600500578CFIA.

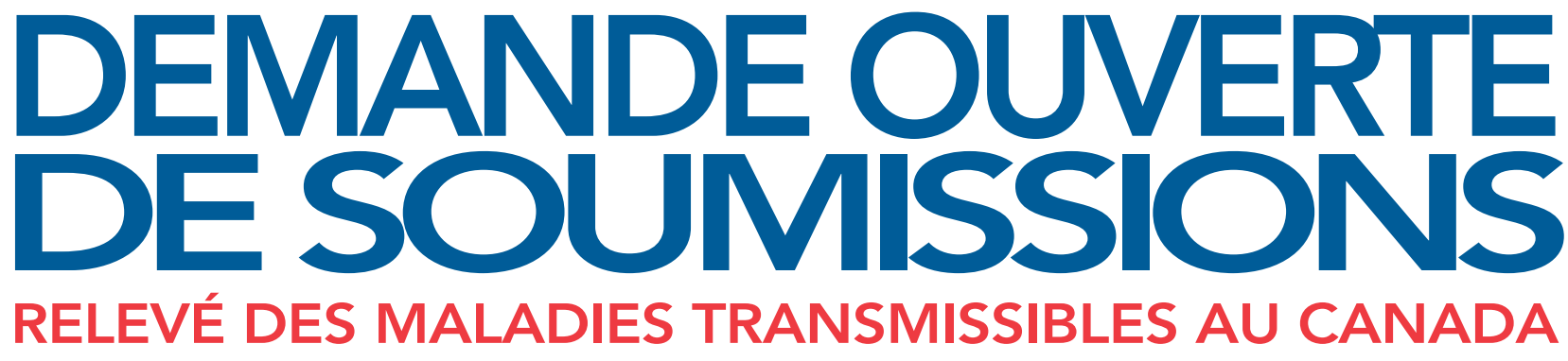

En 2015, nous avons publié des articles d'auteurs provenant de la majorité des provinces et des territoires.

Nous vous invitons à soumettre des articles contenant des renseignements qui font autorité sur les maladies infectieuses, qui éclaireront les politiques, les programmes et les pratiques.

\section{Visitez : www.phac-aspc.gc.ca/publicat/ccdr-rmtc/ia-ra-fra.php

